\title{
Continuous inflorescence removal changes parameters, lignin and saponin a content and alters the transcriptome of root of Bupleurum chinense DC
}

\section{Hui Wang}

Chinese Academy of Medical Sciences \& Peking Union Medical College Institute of Medicinal Plant Development

\section{Gaixia Zhang}

Chinese Academy of Medical Sciences \& Peking Union Medical College Institute of Medicinal Plant Development

\section{Zhihui Gao}

Chinese Academy of Medical Sciences \& Peking Union Medical College Institute of Medicinal Plant Development

\section{Chun Sui}

Chinese Academy of Medical Sciences \& Peking Union Medical College Institute of Medicinal Plant Development

\section{Jianhe Wei ( $\nabla$ wjianh@263.net )}

Chinese Academy of Medical Sciences \& Peking Union Medical College Institute of Medicinal Plant Development

\section{Research article}

Keywords: Bupleurum chinense DC., Continuous inflorescence removal, Root, Transcriptome sequencing, Gene expression pattern

Posted Date: August 11th, 2020

DOI: https://doi.org/10.21203/rs.2.20463/v2

License: (c) (1) This work is licensed under a Creative Commons Attribution 4.0 International License. Read Full License 
The authors have withdrawn this preprint from Research Square 